\title{
Bio-physic constraint model using spatial registration of delta I8F-fluorodeoxyglucose positron emission tomography/computed tomography images for predicting radiation pneumonitis in esophageal squamous cell carcinoma patients receiving neoadjuvant chemoradiation
}

This article was published in the following Dove Press journal: OncoTargets and Therapy

Tien-Chi Hou'

Kun-Yao Dai'

Ming-Che $\mathrm{Wu}^{2}$

Kai-Lung $\mathrm{Hua}^{3}$

Hung-Chi Tai'

Wen-Chien Huang ${ }^{4}$

Yu-Jen Chen ${ }^{1,5}$

'Department of Radiation Oncology, Mackay Memorial Hospital, Taipei, Taiwan; ${ }^{2}$ Department of Nuclear Medicine, Mackay Memorial Hospital, Taipei, Taiwan; ${ }^{3}$ Department of Computer Science and Information Engineering, National Taiwan University of Science and Technology, Taipei, Taiwan; ${ }^{4}$ Department of Surgery, Division of Thoracic Surgery, Mackay Memorial Hospital, Taipei City I0449, Taiwan; ${ }^{5}$ Department of Medical Research, China Medical University Hospital, Taichung 40402, Taiwan

Correspondence: Wen-Chien Huang Department of Surgery, Division of Thoracic Surgery, MacKay Memorial Hospital, No. 92, Sec. 2, Zhongshan N Road, Taipei City 10449, Taiwan Tel +886225433535 Ext I568 Email wjhuang0@yahoo.com.tw

Yu-Jen Chen

Department of Radiation Oncology,

Mackay Memorial Hospital, No. 45,

Minsheng Road, Tamsui District, New

Taipei City 25I60, Taiwan

Tel +88622809 466l Ext 2301

Email chenmdphd@gmail.com
Purpose: This study integrated clinical outcomes and radiomics of advanced thoracic esophageal squamous cell carcinoma patients receiving neoadjuvant concurrent chemoradiotherapy (NACCRT) to establish a novel constraint model for predicting radiation pneumonitis (RP).

Patients and methods: We conducted a retrospective review for thoracic advanced esophageal cancer patients who received NACCRT. From 2013 to 2018, 89 patients were eligible for review. Staging workup and response evaluation included positron emission tomography/computed tomography (PET/CT) scans and endoscopic ultrasound. Patients received RT with 48 Gy to gross tumor and 43.2 Gy to elective nodal area in simultaneous integrated boost method divided in 24 fractions. Weekly platinum-based chemotherapy was administered concurrently. Side effects were evaluated using CTCAE v4. Images of 2-fluoro-2-deoxyglucose PET/CT before and after NACCRT were registered to planning $\mathrm{CT}$ images to create a region of interest for dosimetry parameters that spatially matched RP-related regions, including $\mathrm{V}_{10}, \mathrm{~V}_{20}, \mathrm{~V}_{50 \%}, \mathrm{~V}_{27}$, and $\mathrm{V}_{30}$. Correlation between bio-physic parameters and toxicity was used to establish a constraint model for avoiding RP.

Results: Among the investigated cohort, clinical downstaging, complete pathological response, and 5-year overall survival rates were 59.6\%, 40\%, and $34.4 \%$, respectively. Multivariate logistic regression analysis demonstrated that each individual set standardized uptake value ratios (SUVRs), neither pre- nor post-NACCRT, was not predictive. Interestingly, cutoff increments of $6.2 \%$ and $8.9 \%$ in SUVRs (delta-SUVR) in registered $\mathrm{V}_{20}$ and $\mathrm{V}_{27}$ regions were powerful predictors for acute and chronic RP, respectively.

Conclusion: Spatial registration of metabolic and planning CT images with delta-radiomics analysis using fore-and-aft image sets can establish a unique bio-physic prediction model for avoiding RP in esophageal cancer patients receiving NACCRT.

Keywords: esophageal cancer, neoadjuvant concurrent chemoradiation, radiation pneumonitis, PET/CT, constraint model

\section{Introduction}

Esophageal cancer is the eighth most common cancer, causing about 400,000 deaths worldwide every year. The 5-year overall survival rate for esophageal cancer ranges from $15 \%$ to $25 \%{ }^{1}$ It is difficult to detect early and is usually diagnosed at 
advanced stages. $^{2}$ Adenocarcinoma and squamous cell carcinoma are the main histological types of esophageal cancer with significantly heterogenous distribution between occidental and oriental worlds. ${ }^{2,3}$ Squamous cell carcinoma accounts for more than $90 \%$ of all esophageal cancer cases in the pan-Asia region and appears to have poorer prognoses and earlier lymph node metastasis in comparison to adenocarcinoma. ${ }^{4-6}$ Nearly $50 \%$ of locoregional esophageal cancers are unresectable or borderline resectable disease at diagnosis. ${ }^{7}$ Neoadjuvant treatment, especially chemoradiation, has been shown to increase the $\mathrm{R} 0$ resection rate with acceptable pathological complete response ( $\mathrm{pCR}$ ) ratio and improved survival in locally advanced disease. Therefore, several guidelines recommend neoadjuvant chemoradiation for downstaging before radical surgery. ${ }^{8,9}$ However, subjects of current reported neoadjuvant chemoradiation therapy (NACCRT) trials were recruited from occidental countries and mainly consisted of adenocarcinoma cases. ${ }^{10,11}$ Therefore, conclusions from these studies do not provide strong supporting evidence for squamous cell carcinoma. ${ }^{12}$

Successful radical surgery at 5-8 weeks after completion of NACCRT is critical to the clinical outcome of advanced esophageal cancer patients. ${ }^{13}$ Therefore, development of acute radiation complications, especially radiation pneumonitis (RP), may compromise the prognosis of this multi-modality treatment strategy. ${ }^{14}$ The radiotherapy (RT) field design has to cover gross tumors and the regional lymphatic basin; therefore, it is inevitable to irradiate central parts of the lung adjacent to para-esophageal tissue. As a result, RT may increase the risk of RP, which may impair the success of radical surgery and postoperative pulmonary rehabilitation. ${ }^{15,16}$ Therefore, developing a useful tool for predicting RP is clinically important.

Dose-volume histogram (DVH) generated from RT treatment planning system is widely used as a pre-treatment physic constraint model to evaluate dose distribution for tumor coverage and normal tissue avoidance. Several radiation physic studies demonstrated the role of DVH in predicting the risk of RP. ${ }^{17-19}$ However, DVHbased predictors for RP remain unsatisfactory due to concerns such as having markedly individual variations (such as smoking status, tuberculosis, asthma, chronic obstructive pulmonary disease, and other pulmonary comorbidities), confounding by institutional facilities, and no actual engagement of biological effects. The 2deoxy-2-[fluorine-18] fluoro-D-glucose positron emission tomography integrated with computed tomography $\left({ }^{18} \mathrm{~F}-\mathrm{FDG}\right.$ PET/CT) scan is preferred for the evaluation of treatment responses in esophageal cancer patients after NACCRT. ${ }^{8,9}$ Song et al reported that changes in standardized uptake value (SUV) levels of gross tumors were associated with metabolic responses and pathological complete resection ratios. $^{20}$ Cunliffe et al quantified radiomic changes in serial $\mathrm{CT}$ scans and correlated RT doses to RP incidence. ${ }^{21}$ Recent studies used functional lung heterogeneity in PET/CT imaging before treatment to predict RP among patients receiving definitive thoracic radiation. $^{22,23}$ However, precise and easily used posttreatment radiomics-based predictors of RP are still in development. Given that acute radiation reactions in the lung are a dynamic process and that inflammation can be assessed by dynamic metabolic imaging (before treatment and 5-8 weeks after completion of NACCRT for preoperative survey), it is of interest to explore the combination of CT and metabolic imaging modalities with RT dosimetry parameters to analyze their correlation to RP with regards to spatial registration and delta calculations.

In the present study, we quantified the lung tissue reaction at different RT dose levels and volumes using fore-and-aft ${ }^{18}$ F-FDG PET/CT scan images of patients who received NACCRT for advanced thoracic esophageal cancer. Our goal was to establish a unique and clinically applicable constraint model for RP and lung fibrosis.

\section{Materials and methods}

\section{Patient demographic data and workup}

We conducted a retrospective review of advanced thoracic esophageal cancer patients who received NACCRT in our institution from September 2013 to September 2018. Clinical stages with lymph node positive or $>\mathrm{T} 2$ disease without tissue-proven distant metastasis were eligible for this study. All eligible cohorts were required to have an Eastern Cooperative Oncology Group performance status scale of between 0 and 1. Patients with previous chemotherapy or thoracic RT were excluded. Eighty-eight patients were eligible for assessment of clinical outcome and toxicity. The cancer staging and post-NACCRT surveillances were conducted using CT scan, endoscopic ultrasound with bronchoscope (optional), esophago-gastro-duodenoscopy, and whole body ${ }^{18}$ F-FDG PET/CT. Posttreatment imaging studies, CT or ${ }^{18} \mathrm{~F}$-FDG PET/CT, were assessed 5-8 weeks after the completion of NACCRT, to avoid treatment-related interference. The clinical and pathological staging was determined according to the TNM classification system announced by 
the American Joint Committee on Cancer (seventh edition). Hematological and pulmonary morbidities were graded using the National Cancer Institute's Common Terminology Criteria for Adverse Events version four (CTCAE v4).

\section{RT delivery and chemotherapy administration}

All 89 eligible patients underwent CT simulation in the supine position and were immobilized with alpha cradle ${ }^{\circledR}$ kits (Smithers Medical Products). Planning CT images with 3-mm slice thickness through the entire neck, thorax, and upper abdomen were obtained. Target volume delineation was performed on the eclipse platform in which gross tumor volume (GTV) consisted of the primary tumor and lymphadenopathy as diagnosed by $\mathrm{CT}$, endoscopic ultrasonography, and FDG PET/CT. Two dose levels for the clinical target volume, $\mathrm{CTV}_{\mathrm{High}}$ and $\mathrm{CTV}_{\text {Low }}$, were defined as follows: $\mathrm{CTV}_{\mathrm{High}}$ included the entire GTV with $1 \mathrm{~cm}$ circumferential and 3-4 cm longitudinal expanding margins whereas $\mathrm{CTV}_{\text {Low }}$ covered the regional lymphatic nodal basin. Elective nodal irradiation was delivered to the supraclavicular fossa if the primary tumor or any enlarged lymphadenopathy were in the upper mediastinum, or to the celiac lymphatics if the primary tumor or any lymphadenopathy were close to the esophagogastric junction. Adding both $\mathrm{CTV}_{\mathrm{High}}$ and $\mathrm{CTV}_{\text {Low }}$ with a $0.5 \mathrm{~cm}$ expanding margin in all directions was defined as $\mathrm{PTV}_{\mathrm{High}}$ and PTV $_{\text {Low }}$, respectively. Treatment plans with associated dose maps were generated by Eclipse treatment planning system version 13 (Varian Medical Systems Inc., Palo Alto, CA, USA) or Pinnacle treatment planning system version 9.2 and 10.1 (Philips Healthcare Inc., Andover, MA, USA). The beam lines were 6-MV or 10-MV photon beams, composited by intensity-modulated radiation therapy (IMRT) or Volumetric Arc Therapy planning. The prescribed doses of $\mathrm{PTV}_{\mathrm{High}}$ and $\mathrm{PTV}_{\text {Low }}$ were 48and 43.2 Gy divided into 24 fractions within a simultaneous integrated boost (SIB) method in 85 of 89 patients. Two patients had two-step cone down boost planning instead of SIB that delivered 45 Gy to the PTV Low $_{\text {and }} 48.6$ Gy to the PTV $_{\text {High }}$ with 1.8 Gy per fraction; two patients underwent reduced planning up to 41.4 Gy with 1.8 Gy per fraction to the PTV $\mathrm{Pow}_{\text {Le }}$ area without $\mathrm{PTV}_{\text {High }}$ boost due to concerns regarding a trachea-esophageal fistula at the previous trachea stenting sites. The coverage of $100 \%$ of the prescribed dose volume exceeded $95 \%$ of the targeted PTV while meeting the normal organ constraints and included the following: maximum dose of spinal cord was $<45 \mathrm{~Gy}$, $\mathrm{V}_{20}$ of lung $<30 \%$ of the whole lung, and mean heart dose $<30$ Gy.

All 89 patients underwent concomitant chemoradiotherapy with weekly platinum-based regimens as follows: 1) cisplatin $30 \mathrm{mg} / \mathrm{m}^{2}, 2$ ) carboplatin (AUC 2), or 3) paclitaxel $50 \mathrm{mg} / \mathrm{m}^{2}$ plus either cisplatin $30 \mathrm{mg} / \mathrm{m}^{2}$ or carboplatin (AUC 2).

\section{Defining dose-level volume on registration of PET/CT fusion image}

Radioisotope activity was controlled by a Capintec CRC ${ }^{\circledR}$ 25PET Dose Calibrator. The standard was set as administration of a weight-based injection of ${ }^{18} \mathrm{~F}$-FDG under a 60-min uptake period. PET/CT scan images were acquired on a Discovery ST (GE Healthcare, Waukesha, WI, USA) scanner. PET images within 5 mins per axial field of view were acquired and underwent post-reconstruction with Gaussian filtration.

\section{Image registration and establishment algorithm for candidate predictors of pneumonitis}

Thirty-three patients with applicable pre- and postNACCRT ${ }^{18} \mathrm{~F}$-FDG PET/CT images were eligible for image registration and analysis. All PET/CT scans before and after NACCRT were imported into the Eclipse treatment planning system. Planning CT images were rigidly co-registered to PET/CT images to avoid unwanted deformation of target organs. The original whole lung volume,

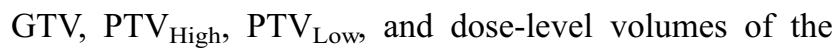
lung $\mathrm{V}_{10}, \mathrm{~V}_{20}, \mathrm{~V}_{27}, \mathrm{~V}_{30}$, and $\mathrm{V}_{50 \%}$ of the maximal prescribed dose, where dose volume $V_{\mathrm{x}}$ was defined as the total organ volume exceeding a radiation dose of " $x$ ", were generated as regions of interest (ROIs) at planning CT and were co-registered to the corresponding relative PET/CT.

Whole lung contouring was automatically generated by the Eclipse treatment planning system (version 13) according to the CT window width between 400 and $2000 \mathrm{HU}$ at the chest, followed by manual removal of the trachea and bilateral main bronchus contours. The accumulated radioactivity concentration, in Bq/c.c., of five different doselevel volume ROIs as described above were calculated using the planning system program and divided by the radioactivity concentration of the whole lung to define the SUVR of each ROI. 


$$
\begin{aligned}
& \text { SUVR of } \mathrm{V}_{x}= \frac{\operatorname{SUV}\left(\mathrm{V}_{\mathrm{x}}\right)}{\mathrm{SUV}(\text { lung })} \\
& \text { Radioactivity } \frac{\text { Concentration of } \mathrm{V}_{\mathrm{x}}\left(\frac{\mathrm{Bq}}{\mathrm{c.c}}\right)}{\text { Radioactivity }} \\
& \begin{array}{l}
\text { Concentration } \\
\text { of reference }(\text { lung })
\end{array}
\end{aligned}
$$

Percentage of delta SUVR ( $\% \Delta$ SUVR) is the relative change of SUVR before and after NACCRT and is described by the following equation:

$$
\% \mathrm{SUVR}=\left(\frac{\text { preTx }_{\mathrm{SUVR}}-{ }_{\text {postTx }} \mathrm{SUVR}}{\text { preTx }_{\mathrm{SUVR}}}\right) \times 100 \%
$$

Percentage of $\mathrm{V}_{\mathrm{x}}$, pre-NACCRT SUVR of $\mathrm{V}_{\mathrm{x}}$, postNACCRT SUVR of $\mathrm{V}_{\mathrm{x}}, \% \Delta$ SUVR of $\mathrm{V}_{\mathrm{x}}$, with $\mathrm{x}$ set to $10 \%, 20 \%, 27 \%, 30 \%$, or $50 \%$ of the $\max$, were evaluated as dosimetric candidate predictors. The dose-level volume at $50 \%$ of the maximum prescription dose $\left(\mathrm{V}_{50 \%}\right)$ served as both a candidate predictor and parameter for the conformality check. The case with V50\% line located out of range of 24-27Gy dose-level lines would consider as extreme value.

\section{Follow up and diagnosis of RP}

The first follow-up was performed 4-8 weeks after the completion of NACCRT and prior to surgery. After surgery, our follow-up policy was modified from the NCCN guideline (Version 2018.2) as follows: if asymptomatic, medical history and physical examination were performed every 3-6 months for 1-2 years, and then every 6-12 months for 3-5 years, followed by annual follow-up. Imaging studies and upper GI endoscopy were performed as clinically indicated. RP was diagnosed through a combination of clinical symptom and radiographic changes. In our institute, RP was considered when the patient presented with non-productive cough, dyspnea, chest pain, and consistent low-grade fever weeks to months after irradiation. Following these findings, arrangements were made for laboratory data collection, chest X-ray, and CT evaluation in order to exclude other possible causes such as infection or tumor recurrence.

\section{Statistical analysis}

Statistical analysis was performed using the Statistical Package for the Social Sciences for Windows, SPSS ${ }^{\circledR}$ software v. 18.0 (IBM Corp., New York, NY, USA; formerly SPSS Inc., Chicago, IL, USA). The comparison of patient characteristics between different grades of RP or chronic radiation lung fibrosis were evaluated using independent $t$-test and chi-square with omnibus test under Cox and Snell $\mathrm{R}$ square model. A two-tailed probability $p$ value of $<0.05$ was considered statistically significant. The association between RP and candidate predictors described above was analyzed by simple logistic regression. Multivariate logistic regression with controlling covariants including age, clinical staging, and position of primary lesion at esophagus were subjected to test the effect of candidate predictors and pneumonitis. Receiveroperating characteristic (ROC) analysis was used to evaluate the discriminative power of pneumonitis predictors to distinguish between patients with and without acute RP $\geq$ grade 2 and chronic radiation pulmonary fibrosis $\geq$ grade 3. Cutoff values for $\%$ delta-SUVR pneumonitis predictors were also assessed by the Youden index (Sensitivity +Specificity-1). Kaplan-Meier analysis was used to calculate overall and progression-free survival, which were determined from the date of biopsy to the date of last follow-up.

\section{Results}

\section{Patient characteristics}

Table 1 shows the characteristics of the 89 patients with advanced thoracic esophageal squamous carcinoma treated with NACCRT. The median age at diagnosis was 62 years old (range, 40-87). The major population of the cohort was clinical stage III $(n=72,80.1 \%)$, cT3 $(n=64,71.9 \%)$, lymph node positive $(\mathrm{n}=84,95.5 \%)$, and histological grade 2 differentiation $(n=59,66.3 \%)$. Primary tumor located at middle esophagus accounted for $53.9 \%$ of the patients $(\mathrm{n}=48)$. The average time interval between NACCRT completion and first posttreatment image surveillance was 6.1 \pm 1.2 weeks (mean \pm STD). The median follow-up time was 32.0 months (range, 1.7-60.8 months).

\section{Treatment compliance and outcomes}

Descriptive statistics for treatment compliance are listed in Table 2. Seventy-eight of $89(87.6 \%)$ patients completed the whole course of chemotherapy, and 82 of 89 (92.1\%) patients completed the RT. Interruption of RT occurred in four patients who developed distant metastasis during treatment, two cases progressed to death during NACCRT. Treatment was discontinued in two elderly patients with intolerable fatigue, and in one patient due to severe nausea and weakness after chemotherapy. The 
Table I Baseline characteristics of patients with advanced thoracic esophageal cancer

\begin{tabular}{|c|c|}
\hline Characteristic & Case $\mathbf{N}(\%)$ \\
\hline Total & 89 \\
\hline Gender (M:F) & $86: 4$ \\
\hline Age & $62.1 \pm 9.2$ \\
\hline \multicolumn{2}{|c|}{ Performance status (ECOG score) } \\
\hline 0 & $49(55.1 \%)$ \\
\hline I & $36(40.4 \%)$ \\
\hline 2 & $4(4.5 \%)$ \\
\hline \multicolumn{2}{|l|}{ Clinical T stage } \\
\hline TI & $3(3.4 \%)$ \\
\hline $\mathrm{T} 2$ & 15 (16.9\%) \\
\hline T3 & $68(76.4 \%)$ \\
\hline $\mathrm{T} 4 \mathrm{a}$ & $3(3.4 \%)$ \\
\hline \multicolumn{2}{|l|}{ Clinical $\mathrm{N}$ stage } \\
\hline No & $4(4.5 \%)$ \\
\hline NI & $36(40.4 \%)$ \\
\hline N2 & 33 (37.1\%) \\
\hline N3 & $16(18.0 \%)$ \\
\hline \multicolumn{2}{|l|}{ Clinical M stage } \\
\hline Mo & 84 (94.4\%) \\
\hline MI & $5(5.6 \%)$ \\
\hline \multicolumn{2}{|l|}{ Clinical stage } \\
\hline IIB & $12(13.5 \%)$ \\
\hline IIIA & $29(32.6 \%)$ \\
\hline IIIB & $25(28.1 \%)$ \\
\hline IIIC & $18(20.2 \%)$ \\
\hline IV & $5(5.6 \%)$ \\
\hline \multicolumn{2}{|l|}{ Differential grade } \\
\hline NA & 17 (19.1\%) \\
\hline 1 & $\mathrm{I}(\mathrm{I} . \mathrm{l} \%)$ \\
\hline 2 & $59(66.3 \%)$ \\
\hline 3 & $12(13.5 \%)$ \\
\hline \multicolumn{2}{|c|}{ Esophagus position } \\
\hline Upper & $21(23.6 \%)$ \\
\hline Middle & 48 (53.9\%) \\
\hline Lower & $20(22.5 \%)$ \\
\hline
\end{tabular}

Note: Data were presented as mean \pm standard deviation in age and case number (percentage) in others.

Abbreviations: ECOG score, Eastern Cooperative Oncology Group performance status score; NA, non-assessment in differential grade due to sample amount of endoscopic-aid biopsy.

$85.4 \%$ (76/89) overall compliance rate indicated favorable tolerance. Table 3 shows the clinical treatment outcomes. The clinical downstaging rate, defined as decreases in
Table 2 Treatment and compliance of patients with advanced thoracic esophageal cancer

\begin{tabular}{|c|c|}
\hline Characteristic & Case N (\%) \\
\hline Treatment duration & $34.1 \pm 6.0$ \\
\hline \multicolumn{2}{|c|}{ Concomitant chemotherapy } \\
\hline Weekly platinum & 48 (53.9\%) \\
\hline Platinum-Taxol & $35(39.3 \%)$ \\
\hline Others & $6(6.8 \%)$ \\
\hline \multicolumn{2}{|c|}{ Chemotherapy dose adjustment } \\
\hline No & $60(67.4 \%)$ \\
\hline Yes & $29(32.6 \%)$ \\
\hline \multicolumn{2}{|c|}{ Chemotherapy completed } \\
\hline Yes & $78(87.6 \%)$ \\
\hline No & II (I2.4\%) \\
\hline \multicolumn{2}{|c|}{ Adjuvant chemotherapy } \\
\hline Yes & 44 (49.4\%) \\
\hline No & $45(50.6 \%)$ \\
\hline \multicolumn{2}{|c|}{ Radiotherapy compliance } \\
\hline Complete & 77 (86.5\%) \\
\hline Modified Fx & $2(2.2 \%)$ \\
\hline Reduction & $3(3.4 \%)$ \\
\hline Incomplete & 7 (7.9\%) \\
\hline \multicolumn{2}{|l|}{ Underwent operation } \\
\hline Yes & $65(73.0 \%)$ \\
\hline No & $24(27.0 \%)$ \\
\hline
\end{tabular}

Notes: Data were presented as mean \pm standard deviation in treatment duration and case number (percentage) in others; weekly platinum, patients underwent weekly cisplatin or weekly carboplatin; platinum-Taxol, patients underwent weekly cisplatin plus paclitaxel or carboplatin plus paclitaxel; modified $\mathrm{Fx}$, prescribed radiotherapy in 180 cGy per fraction upon $48.6 \mathrm{~Gy}$; reduction, planned dose of $\mathrm{CTV}_{\text {High }}$ $<48 \mathrm{~Gy}$.

either $\mathrm{T}$ or $\mathrm{N}$ stage at the first follow-up after NACCRT and prior to surgery, was $59.6 \%$. Subsequently, 65 patients (73\%) were subjected to receive esophagectomy with gastrointestinal tube reconstruction. The pCR rate was $40.0 \%$ (26/65). The 1-, 3-, and 5-year overall survival rates were $62.2 \%, 41.5 \%$, and $34.4 \%$, respectively. The progressionfree survival rate after 1,3 , and 5 years were $46.3 \%$, $23.5 \%$, and $13.9 \%$, respectively. The median overall survival and median progression-free survival were 25.0 months (95\% CI range: 9.7-40.3 months) and 9.4 months (95\% CI range: $3.4-15.5$ months), respectively. The local recurrence rate was $20.0 \%(13 / 65)$ and the distant recurrence rate was $49.2 \%$ (32/65). Kaplan-Meier survival curves of overall survival and progression-free survival are shown in Figure 1. 
Table 3 Clinical outcomes

\begin{tabular}{|l|l|}
\hline Characteristic & N (\%) \\
\hline Response $(\mathrm{N}=89)$ & $29(32.6 \%)$ \\
\hline CR & $24(27.0 \%)$ \\
PR & $12(13.4 \%)$ \\
SD & $24(27.0 \%)$ \\
PD & \\
\hline Excision status $(\mathrm{N}=89)$ & $24(27.0 \%)$ \\
\hline No Operation & $6 I(68.6 \%)$ \\
R0 & $2(2.2 \%)$ \\
RI & $2(2.2 \%)$ \\
R2 & \\
\hline Pathology T stage $\left(\mathrm{N}=65^{\mathrm{a}}\right)$ & $27(41.5 \%)$ \\
\hline T0 & $1(1.5 \%)$ \\
Tis & $7(10.8 \%)$ \\
TI & $14(21.5 \%)$ \\
T2 & $15(23.1 \%)$ \\
T3 & I(I.I\%) \\
T4a, b & $52(80.0 \%)$ \\
\hline Pathology N stage $\left(\mathrm{N}=65^{\mathrm{a}}\right)$ & $5(7.7 \%)$ \\
\hline N0 & $2(3.2 \%)$ \\
NI & \\
N2 & \\
N3 & \\
\hline
\end{tabular}

Notes: Presented data showed treatment outcome of all eligible patients. Data were described as case number (percentage); ${ }^{a} 65$ of 89 patients were eligible for esophagectomy.

\section{Acute and chronic pulmonary toxicity}

Based on favorable compliance, acceptable pCR rate, and survival rates, toxicity profiles were analyzed and are presented in Table 4. Among these patients, serial CT

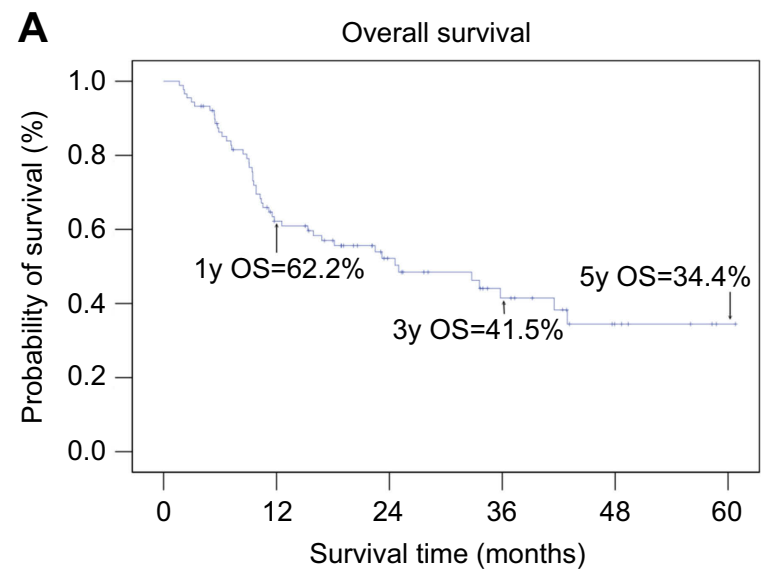

scans from 33 patients having both applicable pre- and post-NACCRT images of ${ }^{18} \mathrm{~F}$-FDG $\mathrm{PET} / \mathrm{CT}$ scan were subjected to review for evaluation of acute and chronic RP. In total, 11 of 33 patients developed grade 2 acute RP. Four of 33 patients (12.2\%) developed moderate to severe acute RP ( $\geq$ grade 3), two of whom died from acute respiratory failure. The above two cases that resulted in mortalities developed severe pulmonary symptoms at 3-4 months after the end of NACCRT, with one case having undergone esophagectomy during this time. For the chronic pulmonary toxicity, five patients had grade 2 pulmonary fibrosis, and required monthly steroid and antitussive treatment for symptom control. Four patients with grade 3 RP developed organic consolidative patches and were hospitalized for repeated occurrence of pneumonia.

\section{Association between clinical and physic parameters and risks of acute and chronic pulmonary toxicity}

Among all clinical parameters, only age was significantly correlated with the incidence of chronic pulmonary fibrosis in univariate logistic regression (hazard ratio $=1.116,95 \% \mathrm{CI}$ $1.008-1.236, p=0.035$ ). Other parameters showed no significant association with lung toxicity. Both simple and multivariate logistic regression analyses demonstrated that all physic parameters, including the DVH, were not associated with statistically significant differences in the incidence of acute RP ( $\geq$ grade 2 ) and chronic pulmonary fibrosis ( $\geq$ grade 3). GTV showed a trend toward chronic radiation pulmonary fibrosis $(p=0.08)$. The results of simple and multivariate logistic regression of physic parameters associated with lung toxicity were described in Tables 5 and 6.

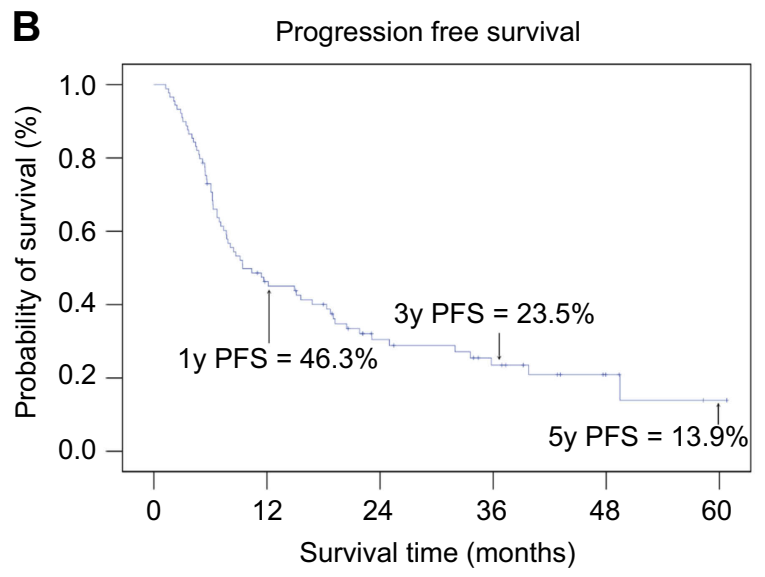

Figure I Kaplan-Meier survival curve of overall survival and progression-free survival. (A) Overall survival (OS) (B) progression-free survival (PFS) of all 89 reviewed patients in presented study at $1,3,5$ years relatively. 
Table 4 Frequency of hematological and pulmonary toxicities

\begin{tabular}{|c|c|c|c|c|}
\hline \multirow[t]{2}{*}{ Characteristic } & \multicolumn{4}{|c|}{ Grade of adverse effect, $\mathbf{N}(\%)$} \\
\hline & 0 & $\mathbf{I}$ & 2 & $\geq 3$ \\
\hline \multicolumn{5}{|l|}{ All patients $(\mathrm{N}=89)$} \\
\hline Anemia & $12(13.5 \%)$ & $29(32.6 \%)$ & $42(47.2 \%)$ & $6(6.7 \%)$ \\
\hline Neutropenia & $28(31.5 \%)$ & $33(37.1 \%)$ & $22(24.7 \%)$ & $6(6.7 \%)$ \\
\hline $\mathrm{RP}$ & $45(50.6 \%)$ & $16(18.0 \%)$ & $22(24.7 \%)$ & $5(6.7 \%)$ \\
\hline \multicolumn{5}{|c|}{ Patients eligible for $\triangle S U V R$ statistic } \\
\hline Acute RP $(\mathrm{N}=33)$ & $12(36.4 \%)$ & $6(18.2 \%)$ & II (33.3\%) & $4(12.2 \%)$ \\
\hline Chronic RP $\left(\mathrm{N}=3 \mathrm{I}^{\mathrm{a}}\right)$ & $19(61.3 \%)$ & $3(9.7 \%)$ & $5(16.1 \%)$ & $4(12.9 \%)$ \\
\hline
\end{tabular}

Notes: Data were described as case number (percentage); grade of adverse effect defined according to Common Terminology Criteria for Adverse Events (CTCAE) version 4.0; ' two of 33 patients died from acute radiation pneumonitis; and therefore, were not counted in the chronic radiation pneumonitis category.

Abbreviations: RP, radiation pneumonitis; $\triangle \mathrm{SUVR}$, fore-and-aft changes of standard uptake value ratio of PET/CT images.

\section{Association between changes of metabolic response imaging and risks of acute and chronic pulmonary toxicity}

ROIs generated according to dose-level volume of described physic parameters on planning $\mathrm{CT}$ images were rigidly co-registered to $\mathrm{PET} / \mathrm{CT}$ images (representative data presented in Figure 2). Percentage of change between pre- and post-NACCRT SUVR was defined and described with $\% \Delta$ SUVR. All $\% \Delta$ SUVR of $V_{x}$ parameters had significant correlation to acute RP in both simple and multivariate regression. For chronic radiation pulmonary fibrosis, both simple and multivariate regression analyses demonstrated a significant correlation between $\% \Delta$ SUVR of $\mathrm{V}_{50 \%}, \mathrm{~V}_{27}$, and $\mathrm{V}_{30}$. By contrast, separate analyses using the pre- or post-NACCRT SUVR parameters independently showed no statistically significant differences between the incidence of acute RP and chronic radiation pulmonary fibrosis in simple and multivariate logistic regression. Tables 5 and 6 display results from the correlation analyses between PET/CT dosimetric parameters and incidence of RP.

\section{Establishment of the bio-physic constraint model for acute and chronic RP}

From the statistically significant multivariant predictors shown in Table 6, the cutoff value maximizing the Youden index to predict patients with high risk of acute and chronic radiation pulmonary toxicity was calculated and is summarized in Table 7. The Youden thresholds corresponded to a cutoff value point with $>90 \%$ sensitivity and $>75 \%$ specificity for prediction of acute RP. Nevertheless, cutoff values for predictors of chronic radiation pulmonary fibrosis corresponded to about $90 \%$ for both sensitivity and specificity. These predictors were above AUC $>0.9$, which indicates good discriminative power. The cutoff value at the $6.2 \%$ increment of $\mathrm{V}_{20}$ area $\triangle \mathrm{SUVR}$ and the $8.9 \%$ increment of $\mathrm{V}_{27}$ area $\triangle \mathrm{SUVR}$ were selected as the new bio-physic constraint. ROC curves for the above two predictors are presented in Figure 3.

\section{Discussion}

This study associated the changes in serial metabolic response imaging to the NACCRT-induced acute and chronic pulmonary toxicity in esophageal cancer. We demonstrated that the increment of SUVR (delta-SUVR) of PET/CT in specific dose-level volumes positively correlated to the incidence of radiation-induced acute RP and chronic lung fibrosis, whereas single time point SUVR or traditional physic parameters, used separately, did not. The cutoff values at the $6.2 \%$ increment of $\mathrm{V}_{20}$ area $\triangle \mathrm{SUVR}$ and the $8.9 \%$ increment of $\mathrm{V}_{27}$ area $\triangle \mathrm{SUVR}$ are powerful predictors of acute RP and chronic lung fibrosis, respectively, and could potentially represent a new bio-physic constraint model in treatment planning for NACCRT in patients with advanced thoracic esophageal squamous cell carcinoma.

In the present study, all physic parameters based on the lung DVH failed to show statistically significant correlation with pulmonary toxicity. Among the study cohorts, only four patients had $\mathrm{V}_{20}$ exceeding 30\%. However, the incidence of RP developed much more frequently than expected, implying that DVH parameters alone may not be the key for predicting RP. During the past decade, radiation oncologists have evaluated thoracic treatment planning according to physic parameters based on DVH. ${ }^{24}$ The QUANTEC board serially 
Table 5 Simple logistic regression for physic and metabolic image parameters

\begin{tabular}{|c|c|c|c|c|c|c|c|}
\hline \multicolumn{2}{|l|}{ Variable } & \multicolumn{3}{|c|}{ Acute radiation pneumonitis grade $\geq 2$} & \multicolumn{3}{|c|}{ Chronic pneumonitis grade $\geq 3$} \\
\hline & & \multirow{5}{*}{$\begin{array}{l}\text { Hazard ratio }(95 \% \mathrm{CI}) \\
0.994(0.982-1.006) \\
0.999(0.998-1.000) \\
0.999(0.997-1.001) \\
0.995(0.990-1.001)\end{array}$} & \multirow{5}{*}{$\begin{array}{l}P \\
0.324 \\
0.193 \\
0.300 \\
0.081\end{array}$} & \multirow{5}{*}{$\begin{array}{l}\mathbf{R}^{2} \\
0.034 \\
0.054 \\
0.036 \\
0.132\end{array}$} & \multirow{5}{*}{$\begin{array}{l}\text { Hazard ratio }(95 \% \mathrm{CI}) \\
1.219(0.898-1.657) \\
0.974(0.944-1.005) \\
0.999(0.998-1.001) \\
0.997(0.993-1.001)\end{array}$} & \multirow{5}{*}{$\begin{array}{l}p \\
0.095 \\
0.345 \\
0.168 \\
0.144\end{array}$} & \multirow{5}{*}{$\begin{array}{l}\mathbf{R}^{\mathbf{2}} \\
0.160 \\
0.030 \\
0.103 \\
0.103\end{array}$} \\
\hline Volume & Tumor & & & & & & \\
\hline & Lung & & & & & & \\
\hline & PTV $_{\text {Low }}$ & & & & & & \\
\hline & PTV $_{\text {High }}$ & & & & & & \\
\hline \multirow[t]{5}{*}{ Lung DVH } & $V_{10}$ & $0.995(0.946-1.046)$ & 0.838 & 0.995 & $0.995(0.989-1.002)$ & 0.728 & 0.004 \\
\hline & $V_{20}$ & $1.039(0.930-1.160)$ & 0.500 & 0.014 & $0.990(0.934-1.049)$ & 0.855 & 0.001 \\
\hline & $V_{50 \%}$ & $1.061(0.925-1.217)$ & 0.397 & 0.022 & $1.012(0.892-1.147)$ & 0.469 & 0.017 \\
\hline & $V_{27}$ & $1.049(0.911-1.208)$ & 0.505 & 0.014 & $1.060(0.905-1.24 I)$ & 0.435 & 0.020 \\
\hline & $V_{30}$ & $1.053(0.904-1.227)$ & 0.507 & 0.013 & $1.068(0.906-1.258)$ & 0.345 & 0.030 \\
\hline \multirow[t]{8}{*}{ Pretreatment SUVR } & $V_{10}$ & $0.004(0.000-21.84)$ & 0.211 & 0.052 & $1.090(0.911-1.303)$ & 0.169 & 0.073 \\
\hline & $V_{20}$ & $0.035(0.000-9.483)$ & 0.240 & 0.044 & $0.001(0.000-24.85)$ & 0.081 & 0.116 \\
\hline & $V_{50 \%}$ & $0.063(0.000-8.625)$ & 0.271 & 0.038 & $0.001(0.000-2.252)$ & 0.159 & 0.072 \\
\hline & $V_{27}$ & $0.131(0.002-8.972)$ & 0.131 & 0.029 & $0.012(0.000-5.631)$ & 0.157 & 0.076 \\
\hline & $V_{30}$ & $0.268(0.004-15.91)$ & 0.268 & 0.012 & $0.017(0.000-4.843)$ & 0.195 & 0.060 \\
\hline & GTV & $0.975(0.820-1.158)$ & 0.770 & 0.003 & $0.033(0.000-5.755)$ & 0.837 & 0.001 \\
\hline & PTV $_{\text {Low }}$ & $0.858(0.373-1.970)$ & 0.718 & 0.004 & $0.980(0.806-1.190)$ & 0.606 & 0.009 \\
\hline & PTV $_{\text {High }}$ & $0.789(0.5 \mid 8-1.202)$ & 0.789 & 0.039 & $0.773(0.292-2.052)$ & 0.676 & 0.006 \\
\hline \multirow[t]{8}{*}{ Posttreatment SUVR } & $V_{10}$ & $459.5(0.895-2.360 \mathrm{E}+5)$ & 0.054 & 0.149 & $1.098(0.709-1.700)$ & 0.070 & 0.137 \\
\hline & $V_{20}$ & $46.4 \mathrm{I}(0.824-2.6 \mathrm{I} 2 \mathrm{E}+4)$ & 0.062 & 0.153 & $496.3(0.595-4.138 \mathrm{E}+5)$ & 0.098 & 0.115 \\
\hline & $V_{50 \%}$ & $33.36(0.709-1.568 \mathrm{E}+3)$ & 0.074 & 0.159 & $21.37(0.57 \mid-800.0)$ & 0.120 & 0.130 \\
\hline & $V_{27}$ & $37.03(0.872-1.573 E+3)$ & 0.059 & 0.172 & I4.34 (0.50I-4I0.5) & 0.117 & 0.124 \\
\hline & $V_{30}$ & $33.82(0.899-1.273 E+3)$ & 0.057 & 0.178 & $12.06(0.536-27 \mid .3)$ & 0.125 & 0.125 \\
\hline & GTV & $1.710(0.836-3.501)$ & 0.142 & 0.073 & $10.10(0.528-193.2)$ & 0.058 & 0.136 \\
\hline & PTV $_{\text {Low }}$ & $1.872(0.462-7.586)$ & 0.380 & 0.024 & $2.236(0.972-5.140)$ & 0.597 & 0.009 \\
\hline & PTV $_{\text {High }}$ & $0.833(0.392-1.769)$ & 0.634 & 0.007 & I.545 (0.308-7.745) & 0.180 & 0.073 \\
\hline \multirow[t]{8}{*}{ \%Delta-SUVR } & $V_{10}$ & $1.709(1.181-2.474)$ & $0.004 *$ & 0.557 & $2.265(0.686-7.47 I)$ & 0.074 & 0.602 \\
\hline & $\mathrm{V}_{20}$ & $1.736(1.098-2.744)$ & $0.018^{*}$ & 0.572 & $2.965(0.900-9.774)$ & 0.308 & 0.657 \\
\hline & $V_{50 \%}$ & $1.273(1.056-1.534)$ & $0.01 I^{*}$ & 0.459 & $6.872(0.169-279.5)$ & $0.013^{*}$ & 0.448 \\
\hline & $V_{27}$ & $1.248(1.046-1.488)$ & $0.014^{*}$ & 0.448 & $1.299(1.057-1.596)$ & $0.015^{*}$ & $0.44 I$ \\
\hline & $V_{30}$ & I.191 (1.033-1.373) & $0.016 *$ & 0.401 & 1.277 (I.049-I.554) & $0.020 *$ & 0.403 \\
\hline & GTV & $1.010(0.98 \mid-1.04 I)$ & 0.496 & 0.014 & $1.213(1.030-1.427)$ & 0.150 & 0.067 \\
\hline & PTV $_{\text {Low }}$ & $1.012(0.978-1.048)$ & 0.486 & 0.015 & $1.025(0.991-1.059)$ & 0.374 & 0.026 \\
\hline & PTV $_{\text {High }}$ & $1.013(0.979-1.048)$ & 0.453 & 0.018 & $1.018(0.979-1.058)$ & 0.305 & 0.037 \\
\hline
\end{tabular}

Notes: Presented data showed the correlation coefficient at simple logistic regression for all the physic and bio-physic parameters; ${ }^{*}$ marked the statistical significance with $p$-value $<0.05$.

Abbreviations: DVH, Dose Volume Histogram; $R^{2}$, pseudo- $R$ square value estimated with Cox and Snell method; GTV, Gross Target Volume; $V_{x}$, total organ volume exceeding a radiation dose of " $x$ "; SUVR, Relative Standard Uptake Value.

reported advise of diametric constraints based on conclusions that the mean lung dose and $\mathrm{V}_{20}$ were best supported for routine clinical practice. The latest statement released in 2010 recommended maintaining the $\mathrm{V}_{20}$ at $<30-35 \%$ and mean lung dose between 20 and 23 Gy in order to lower the risk of pneumonitis to $<20 \%{ }^{25}$ However, the association between calculated dosimetry and normal organ outcome was estimated mainly using results of definitive RT and 3Dconformal RT planning, which may present difficulty in interpreting toxicity of multimodality treatment when using CCRT and IMRT. ${ }^{26,27}$ Several chemotherapy agents are known to be radiation sensitizers and therefore increase the risk of radiation reactions, including lung injury. A meta-analysis demonstrated a 1.6-fold increase in hazard ratio of RP in patients receiving CCRT compared to those receiving sequential regimen. ${ }^{28}$ When multimodality treatments consisted of RT and concurrent administration of chemotherapeutics, targeted agents, or immunotherapy, the establishment of predictors using a practical constraint model to avoid RP remains an urgent task. 
Table 6 Multivariate logistic regression for physic and metabolic image parameters

\begin{tabular}{|c|c|c|c|c|c|c|c|}
\hline \multicolumn{2}{|l|}{ Variable } & \multicolumn{3}{|c|}{ Acute radiation pneumonitis Grade $\geq 2$} & \multicolumn{3}{|c|}{ Chronic pneumonitis Grade $\geq 3$} \\
\hline & & \multirow{5}{*}{$\begin{array}{l}\text { Hazard ratio }(95 \% \mathrm{Cl}) \\
1.001(0.986-1.017) \\
1.000(0.998-1.001) \\
0.999(0.997-1.002) \\
0.996(0.989-1.003)\end{array}$} & \multirow{5}{*}{$\begin{array}{l}p \\
0.856 \\
0.472 \\
0.717 \\
0.284\end{array}$} & \multirow{5}{*}{$\begin{array}{l}\mathbf{R}^{2} \\
0.207 \\
0.219 \\
0.209 \\
0.286\end{array}$} & \multirow{5}{*}{$\begin{array}{l}\text { Hazard ratio }(95 \% \mathrm{CI}) \\
0.986(0.95 \mathrm{I}-\mathrm{I} .02 \mathrm{I}) \\
\mathrm{I} .000(0.998-1.00 \mathrm{I}) \\
\mathrm{I} .000(0.998-\mathrm{I} .00 \mathrm{I}) \\
0.996(0.988-\mathrm{I} .005)\end{array}$} & \multirow{5}{*}{$\begin{array}{l}p \\
0.427 \\
0.645 \\
0.645 \\
0.395\end{array}$} & \multirow{5}{*}{$\begin{array}{l}\mathbf{R}^{\mathbf{2}} \\
0.251 \\
0.237 \\
0.251 \\
0.249\end{array}$} \\
\hline Volume & Tumor & & & & & & \\
\hline & Lung & & & & & & \\
\hline & PTV $_{\text {Low }}$ & & & & & & \\
\hline & PTV $_{\text {High }}$ & & & & & & \\
\hline \multirow[t]{5}{*}{ Lung DVH } & $V_{10}$ & $1.008(0.947-1.072)$ & 0.811 & 0.207 & $1.011(0.937-1.090)$ & 0.784 & 0.234 \\
\hline & $\mathrm{V}_{20}$ & $1.072(0.935-1.228)$ & 0.319 & 0.230 & $1.028(0.875-1.207)$ & 0.740 & 0.235 \\
\hline & $V_{50 \%}$ & $1.087(0.914-1.292)$ & 0.348 & 0.228 & $1.070(0.876-1.307)$ & 0.506 & 0.243 \\
\hline & $V_{27}$ & $1.068(0.892-1.278)$ & 0.473 & 0.219 & $1.076(0.870-1.330)$ & 0.502 & 0.244 \\
\hline & $V_{30}$ & $1.069(0.878-1.302)$ & 0.508 & 0.217 & $1.112(0.873-1.417)$ & 0.391 & 0.251 \\
\hline \multirow[t]{8}{*}{ Pretreatment SUVR } & $V_{10}$ & $0.000(0.000-8.618)$ & 0.117 & 0.270 & $0.000(0.000-498.4)$ & 0.256 & 0.270 \\
\hline & $V_{20}$ & $0.012(0.000-12.86)$ & 0.215 & 0.245 & $0.002(0.000-8.877)$ & 0.148 & 0.289 \\
\hline & $V_{50 \%}$ & 0.07 I (0.000-28.73) & 0.388 & 0.224 & $0.059(0.000-76.02)$ & 0.439 & 0.247 \\
\hline & $V_{27}$ & $0.202(0.00 \mathrm{I}-38.06)$ & 0.550 & 0.215 & $0.093(0.000-76.18)$ & 0.488 & 0.244 \\
\hline & $\mathrm{V}_{30}$ & $0.672(0.004-112.2)$ & 0.879 & 0.207 & $0.208(0.000-99.01)$ & 0.617 & 0.238 \\
\hline & GTV & $0.969(0.789-1.190)$ & 0.764 & 0.208 & $0.992(0.773-1.272)$ & 0.948 & 0.232 \\
\hline & $\operatorname{PTV}_{L} O_{w}$ & 1.017 (0.373-2.773) & 0.974 & 0.206 & $1.041(0.280-3.874)$ & 0.952 & 0.232 \\
\hline & PTV $_{\text {High }}$ & 0.787 (0.477-I.299) & 0.349 & 0.228 & $1.375(0.691-2.737)$ & 0.365 & 0.253 \\
\hline \multirow[t]{8}{*}{ Postreatment SUVR } & $V_{10}$ & $246.1(0.101-5.995 E+5)$ & 0.166 & 0.263 & $4.093 \mathrm{E}+4(0.26 \mathrm{I}-6.418 \mathrm{E}+9)$ & 0.082 & 0.339 \\
\hline & $v_{20}$ & $29.15(0.242-3.516 \mathrm{E}+3)$ & 0.168 & 0.268 & $42.96(0.179-1.034 \mathrm{E}+4)$ & 0.179 & 0.295 \\
\hline & $V_{50 \%}$ & $27.72(0.329-2.337 E+3)$ & 0.142 & 0.280 & $28.24(0.246-3.245 E+3)$ & 0.168 & 0.302 \\
\hline & $V_{27}$ & $40.50(0.468-0.3503 E+3)$ & 0.104 & 0.296 & $28.90(0.274-3.047 E+3)$ & 0.157 & 0.304 \\
\hline & $\mathrm{V}_{30}$ & $43.01(0.556-3.329 E+3)$ & 0.090 & 0.306 & $23.87(0.300-1.898 \mathrm{E}+3)$ & 0.155 & 0.306 \\
\hline & GTV & $1.240(0.514-2.993)$ & 0.632 & 0.212 & $1.612(0.608-4.275)$ & 0.338 & 0.256 \\
\hline & PTV $_{\text {Low }}$ & $0.852(0.159-4.567)$ & 0.852 & 0.207 & $0.678(0.084-5.504)$ & 0.716 & 0.235 \\
\hline & PTV & $0.406(0.136-1.214)$ & 0.107 & 0.276 & $1.321(0.367-4.760)$ & 0.670 & 0.237 \\
\hline \multirow[t]{8}{*}{ \%Delta-SUVR } & $V_{10}$ & 2.077 (1.077-4.008) & $0.029 *$ & 0.591 & $13.50(0.155-1.173 E+3)$ & 0.253 & 0.648 \\
\hline & $V_{20}$ & $1.715(1.088-2.704)$ & $0.020 *$ & 0.584 & $2.934 \mathrm{E}+3(0.000-\mathrm{A} 74 \mathrm{~F})$ & 0.992 & 0.700 \\
\hline & $V_{50 \%}$ & $1.270(1.04|-| .550)$ & $0.019 *$ & 0.483 & $1.262(1.019-1.561)$ & $0.033^{*}$ & 0.478 \\
\hline & $V_{27}$ & $1.247(1.032-1.506)$ & $0.022^{*}$ & 0.476 & $1.242(1.015-1.519)$ & $0.035^{*}$ & $0.47 \mid$ \\
\hline & $V_{30}$ & $1.185(1.016-1.381)$ & $0.03 I^{*}$ & 0.432 & I.177 (1.002-I.382) & $0.047^{*}$ & 0.438 \\
\hline & GTV & $1.009(0.974-1.045)$ & 0.611 & 0.212 & $1.025(0.983-1.068)$ & 0.250 & 0.265 \\
\hline & PTV $_{\text {Low }}$ & $0.992(0.950-1.037)$ & 0.733 & 0.209 & $0.998(0.948-1.051)$ & 0.937 & 0.232 \\
\hline & PTV & $0.984(0.937-1.034)$ & 0.526 & 0.264 & 1.001 (0.952-I.053) & 0.967 & 0.228 \\
\hline
\end{tabular}

Notes: Presented data showed the correlation coefficient at multivariate logistic regression for all physic and bio-physic parameters; *, marked the statistical significance with $p$-value $<0.05$.

Abbreviations: $R^{2}$, pseudo- $R$ square value estimated with Cox and Snell method; $V_{x}$, total organ volume exceeding a radiation dose of " $x$ ".

As a widely used metabolic imaging method, PET/CT may provide a better tool in interpreting biological effects. ${ }^{29}$ Nonetheless, previous studies based on single time point PET scans, either during pretreatment or posttreatment, reported moderate or unsatisfactory predictive power. ${ }^{22,23,30}$ Zhang et al showed a correlation between mean lung SUV and $\mathrm{RP}$ ( $\geq$ grade 2 ) in a group of patients whose radiation dose was more than $60 \mathrm{~Gy}$; however, in a patient group that received $<60$ Gy, statistically significant correlations were not achieved. ${ }^{30}$ Also, their study had a limited power of establishing clear cutoff values for mean lung SUV in predicting RP due to a limited number of cases and limited discriminative capability of the selected parameters. Two studies based on pre-radiation PET scan images established their own predictors with significant correlation to the RP incidence with moderate sensitivity and lower specificity. ${ }^{22,23}$ Lee et al most recently successfully established strong predictors of RP using functional lung heterogenicity and dosimetry parameters using pretreatment perfusion SPECT/CT and FDG PET/CT. ${ }^{31}$ The functional lung heterogenicity describing the preexisting area of 

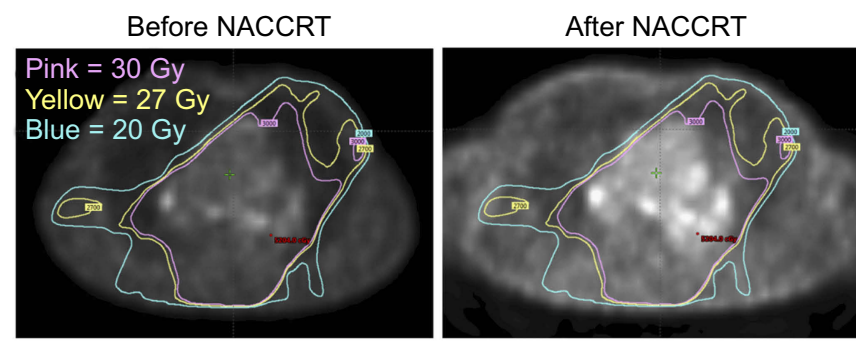

Acute radiation pneumonitis
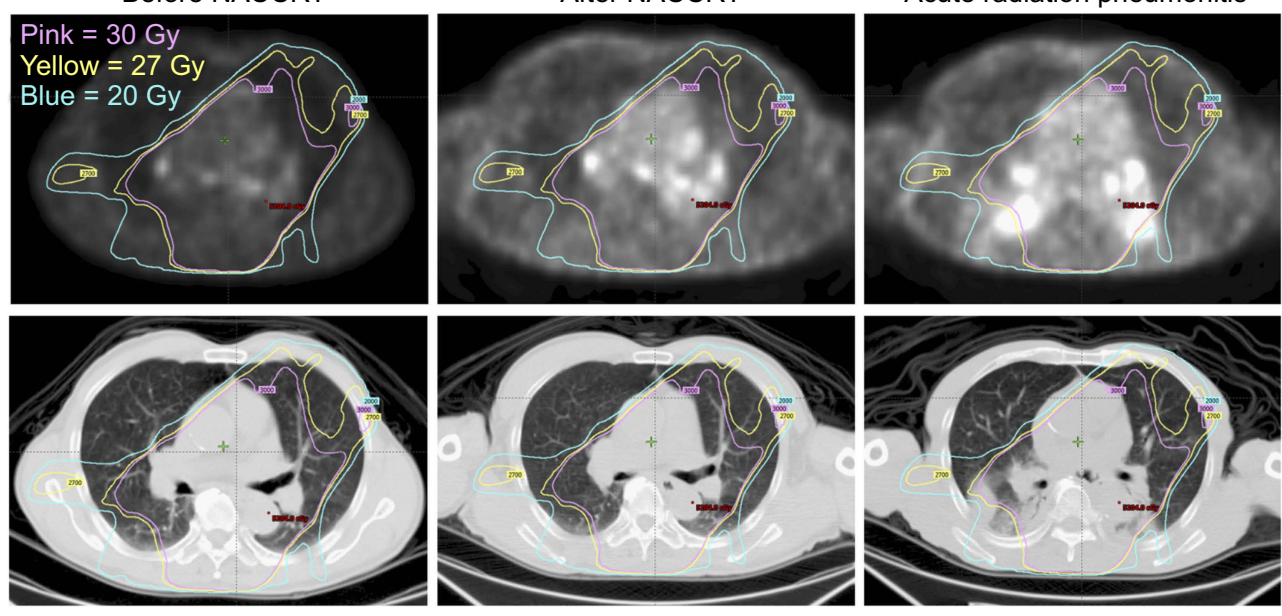

Figure 2 Representative image of patient with grade 3 acute radiation pneumonitis predicted by presented bio-physic parameters. Upper panel showed the PET-dosevolume fusion images. Lower panel showed the CT-dose-volume fusion images; blue, yellow, and pink line circled the volume of prescribed dose exceeding 20,27 , and $30 \mathrm{~Gy}$ relatively.

Table 7 Receiver-operator characteristic operating point of acute radiation pneumonitis and chronic pulmonary fibrosis predictors that maximized the Youden index

\begin{tabular}{|c|c|c|c|c|}
\hline Parameter & $\begin{array}{l}\text { Cutoff } \\
\text { value }\end{array}$ & AUC & Sensitivity & Specificity \\
\hline \multicolumn{5}{|c|}{ Acute radiation pneumonitis ( $\geq$ Gr. 3 ) } \\
\hline $\begin{array}{l}\% \Delta S U V R \text { of } \\
V_{10}\end{array}$ & $+1.5 \%$ & 0.956 & $100 \%$ & $88.9 \%$ \\
\hline $\begin{array}{l}\% \Delta S U V R \text { of } \\
V_{20}\end{array}$ & $+6.2 \%$ & 0.956 & $93.3 \%$ & $88.9 \%$ \\
\hline $\begin{array}{l}\% \Delta S U V R \text { of } \\
V_{50 \%}\end{array}$ & $+5.8 \%$ & 0.926 & $100 \%$ & $83.3 \%$ \\
\hline $\begin{array}{l}\% \Delta S U V R \text { of } \\
V_{27}\end{array}$ & $+4.5 \%$ & 0.926 & $100 \%$ & $88.9 \%$ \\
\hline $\begin{array}{l}\% \Delta S U V R \text { of } \\
V_{30}\end{array}$ & $+5.2 \%$ & 0.911 & $100 \%$ & $77.8 \%$ \\
\hline \multicolumn{5}{|c|}{ Chronic pulmonary fibrosis ( $\geq$ Gr. 2 ) } \\
\hline $\begin{array}{l}\% \Delta S U V R \text { of } \\
V_{50 \%}\end{array}$ & $+8.9 \%$ & 0.929 & $88.9 \%$ & $90.9 \%$ \\
\hline $\begin{array}{l}\% \Delta S U V R \text { of } \\
V_{27}\end{array}$ & $+8.9 \%$ & 0.922 & $88.9 \%$ & $90.9 \%$ \\
\hline $\begin{array}{l}\% \Delta S U V R \text { of } \\
V_{30}\end{array}$ & $+8.9 \%$ & 0.924 & $88.9 \%$ & $90.9 \%$ \\
\hline
\end{tabular}

Abbreviations: AUC, area under the ROC curve; \% $\triangle$ SUVR of $V_{x}$, percentage of SUV change between pre- and post-NACCRT at dose volume $V_{x} ; V_{x}$, total organ volume exceeding a radiation dose of " $x$."

emphysematous lung indicated a potentially hypersensitive microenvironment that may be at increased risk of radiation injury. In agreement with our study, Lee et al found dosefunction histogram parameters to be competent predictors of
RP that were better than other physic parameters. It is important to realize that predictors solely based on pretreatment functional images may not thoroughly represent the dynamic changes in $3 \mathrm{D}$ anatomical features, clinical lung conditions, image textures, and adaptive RT parameters before application in clinical RT. Few studies have mentioned developing serial PET scan imaging for use as RP predictors. A recent study found a relationship between maximal SUV in irradiated lung parenchyma and RP. ${ }^{32}$ Still, the authors only focused on the discrimination of observed phenomena without quantifying the correlation using statistic tools. Also, maximal SUV may not be a precise parameter since lung parenchyma, consisting of bronchial trees and pulmonary vasculatures, has distinct inhomogeneity, which interferes with FDG uptake values of $\mathrm{PET} / \mathrm{CT}$ scans. Considering that acute RP is a dynamic process with inflammatory responses in radiation fields, our bio-physic constraint model using spatial registration and delta calculation algorithms, rather than pre- or post-CCRT image sets alone, could be a practical way to establish specific constraint models for RP across institutions. This model may play a role in developing a model for validation of previous and future RP constraints. In real-world clinical practice, the prediction model indicated that medical physics should eliminate 20and 27 Gy distribution area at lung in treatment planning in esophageal cancer patients going to receive NACCRT. Our algorithm may potentially assist surgeons in evaluating pulmonary morbidity prior to surgery. Additionally, for patients who are predicted to be at high risk of pulmonary morbidity, intensive lung function monitoring and prophylactic steroid administration may be helpful. For proton therapy, the radiobiology of the 
A

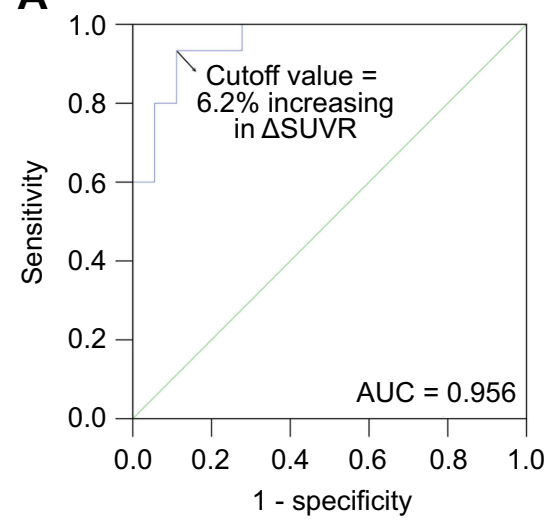

B

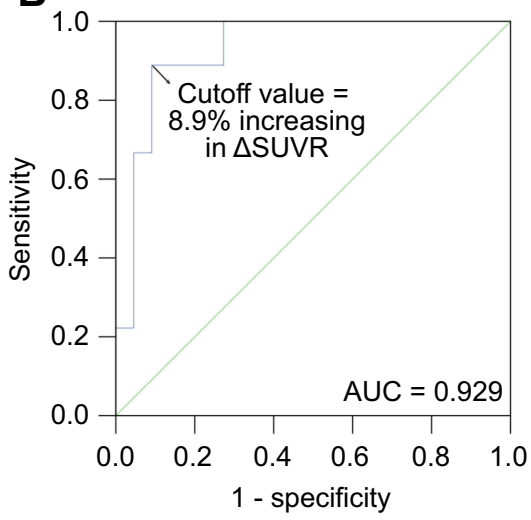

Figure 3 Receiver-operating characteristic (ROC) curves for predictors of the presented bio-physic constraints for acute and chronic radiation pneumonitis (A) acute

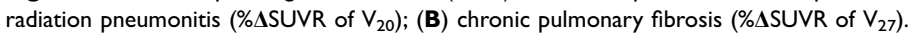

Abbreviations: AUC, area under the ROC curve; \% $\triangle S U V R$ of $V_{x}$, percentage of SUV change between pre- and post-NACCRT at dose volume $V_{x}$.

lung remains unclear, ${ }^{33}$ and as such our model may be used as a reference for establishing new RP constraints for particle therapy. Moreover, predictors based on the concept of this study may better predict RP risks of combination modalities consisting of RT and immune therapy or targeted therapy, compared to physic and dosimetric parameters. The delta model could serve as a platform basis for further incorporation of data from radiomics and genomics.

Although the delta algorithm requires fewer cases, the case numbers used in our study remains insufficient and our results still require further validation. The ${ }^{18} \mathrm{~F}-\mathrm{FDG}$ $\mathrm{PET} / \mathrm{CT}$ has been routinely applied in staging workup and response evaluation for esophageal cancer patients in our institution over the last 3 years but has not yet fully covered all esophageal cancer patients. These retrospective cohorts came from the time points determined for routine use of fore-and-aft PET/CT images, raising the concern of potential selection bias.

\section{Conclusion}

By using this bio-physic constraint model, the specified \% $\triangle$ SUVR of pre- and post-NACCRT PET/CT can potentially be a predictor for acute RP and chronic pulmonary fibrosis in patients with advanced esophageal cancer.

\section{Ethics approval and informed consent}

The study protocol was granted institutional review board approval (serial number: 18MMHIS194e, MacKay Memorial Hospital, Taipei, Taiwan). Written informed consent was obtained from all study participants and research was conducted in accordance with the 1975 Declaration of Helsinki, as revised in 1983.

\section{Acknowledgment}

This study was funded by MacKay Memorial Hospital (grant number: MMH-E-107-13) and the Ministry of Science and Technology, Taiwan (grant number: MOST 106-2314-B-195-002-MY3 and MOST-106-2623-E-195001-NU). The preliminary data of this manuscript has been presented in 2017 ASTRO Annual Meeting, which was held on September 24-27, 2017, at the San Diego Convention Center, San Diego, CA. It has not been published or presented elsewhere in part or in entirety and is not under consideration by another journal. All study participants provided informed consent, and the study design was approved by the appropriate ethics review board. We have read and understood the journals policies, and we believe that neither the manuscript nor the study violates any of these.

\section{Author contributions}

Tien-Chi Hou and Yu-Jen Chen conceived, designed, discussed the study and wrote the manuscript. Tien-Chi Hou and Kun-Yao Dai did the patient follow-up and data analysis. Ming-Che Wu prepared all the PET/CT fusion and registration images and afford all the nuclear medicine technique among the study. Wen-Chien Huang did the endoscopy and biopsy during patient follow-up. Hung-Chi Tai did the calibration of radiation physic parameters and reviewed the treatment planning of radiotherapy. Kai-Long Hua afforded the algorithm of image processing. Yu-Jen Chen and Wen-Chien Huang revised and supervised the study. All authors contributed to 
data analysis, drafting or revising the article, gave final approval of the version to be published, and agree to be accountable for all aspects of the work.

\section{Disclosure}

The authors report no conflicts of interests in this work.

\section{References}

1. Gupta B, Kumar N. Worldwide incidence, mortality and time trends for cancer of the oesophagus. Eur J Cancer Prev. 2017;26(2):107118. doi:10.1097/CEJ.0000000000000249

2. Domper Arnal MJ, Ferrandez Arenas A, Lanas Arbeloa A. Esophageal cancer: risk factors, screening and endoscopic treatment in Western and Eastern countries. World J Gastroenterol. 2015;21 (26):7933-7943. doi:10.3748/wjg.v21.i10.2937

3. Trivers KF, Sabatino SA, Stewart SL. Trends in esophageal cancer incidence by histology, United States, 1998-2003. Int J Cancer. 2008;123(6):1422-1428. doi:10.1002/ijc.v123:6

4. Szumilo J. [Epidemiology and risk factors of the esophageal squamous cell carcinoma]. Pol Merkur Lekarski. 2009;26(151):82-85.

5. Xi M, Xu C, Liao Z, et al. The impact of histology on recurrence patterns in esophageal cancer treated with definitive chemoradiotherapy. Radiother Oncol. 2017;124(2):318-324. doi:10.1016/j.radonc.2017.06.019

6. Bollschweiler E, Holscher AH. Prognosis of early esophageal cancer: differences between squamous cell carcinoma and adenocarcinoma. Ann Surg. 2007;245(2):334. doi:10.1097/01.sla.0000253072.33351.a4

7. Enzinger PC, Mayer RJ. Esophageal cancer. $N$ Engl J Med. 2003;349 (23):2241-2252. doi:10.1056/NEJMoa030969

8. Network TNCC. NCCN clinical practice guidelines in oncology (NCCN guidelines) - esophageal and esophagogastric junction cancers. 2018; Version 2. Available from: https://www.nccn.org/profes sionals/physician_gls/pdf/esophageal.pdf.. Accessed July 12, 2019.

9. Lordick F, Mariette C, Haustermans K, Obermannova R, Arnold D, Committee EG. Oesophageal cancer: ESMO clinical practice guidelines for diagnosis, treatment and follow-up. Ann Oncol. 2016;27 (suppl 5):v50-v57.

10. Rath P, Fahrig A. [Active surveillance and postponed operation after neoadjuvant radiochemotherapy for oesophageal cancer-findings of the preSANO study]. Strahlenther Onkol. 2018. doi:10.1007/s00066018-1371-6.

11. Shapiro J, van Lanschot JJB, Hulshof M, et al. Neoadjuvant chemoradiotherapy plus surgery versus surgery alone for oesophageal or junctional cancer (CROSS): long-term results of a randomised controlled trial. Lancet Oncol. 2015;16(9):1090-1098. doi:10.1016/ S1470-2045(15)00040-6

12. Tsukada Y, Higashi T, Shimada H, Kikuchi Y, Terahara A. The use of neoadjuvant therapy for resectable locally advanced thoracic esophageal squamous cell carcinoma in an analysis of 5016 patients from 305 designated cancer care hospitals in Japan. Int J Clin Oncol. 2018;23(1):81-91. doi:10.1007/s10147-017-1178-y

13. Rizvi FH, Syed AA, Khattak S, Rizvi SS, Kazmi SA, Khan MQ. Complete pathological response after neoadjuvant treatment in locally advanced esophageal cancer predicts long term survival: a retrospective cohort study. Int J Surg. 2014;12(6):621-625. doi:10.1016/j.ijsu.2014.04.014

14. Hamai Y, Hihara J, Taomoto J, Yamakita I, Ibuki Y, Okada M. Effects of neoadjuvant chemoradiotherapy on postoperative morbidity and mortality associated with esophageal cancer. Dis Esophagus. 2015;28 (4):358-364. doi:10.1111/dote.12207
15. Graves PR, Siddiqui F, Anscher MS, Movsas B. Radiation pulmonary toxicity: from mechanisms to management. Semin Radiat Oncol. 2010;20(3):201-207. doi:10.1016/j.semradonc.2010.01.010

16. Morota M, Gomi K, Kozuka T, et al. Late toxicity after definitive concurrent chemoradiotherapy for thoracic esophageal carcinoma. Int J Radiat Oncol Biol Phys. 2009;75(1):122-128. doi:10.1016/j. ijrobp.2008.10.075

17. Zhao Y, Chen L, Zhang S, et al. Predictive factors for acute radiation pneumonitis in postoperative intensity modulated radiation therapy and volumetric modulated arc therapy of esophageal cancer. Thorac Cancer. 2015;6(1):49-57. doi:10.1111/1759-7714.12142

18. Shen WB, Zhu SC, Gao HM, et al. [Low dose volume histogram analysis of the lungs in prediction of acute radiation pneumonitis in patients with esophageal cancer treated with three-dimensional conformal radiotherapy]. Zhonghua Zhong Liu Za Zhi. 2013;35(1):4549.

19. Kumar G, Rawat S, Puri A, et al. Analysis of dose-volume parameters predicting radiation pneumonitis in patients with esophageal cancer treated with 3D-conformal radiation therapy or IMRT. Jpn $J$ Radiol. 2012;30(1):18-24. doi:10.1007/s11604-011-0002-2

20. Song SY, Kim JH, Ryu JS, et al. FDG-PET in the prediction of pathologic response after neoadjuvant chemoradiotherapy in locally advanced, resectable esophageal cancer. Int $J$ Radiat Oncol Biol Phys. 2005;63(4):1053-1059. doi:10.1016/j.ijrobp.2005.03.033

21. Cunliffe A, Armato SG 3rd, Castillo R, Pham N, Guerrero T, AlHallaq HA. Lung texture in serial thoracic computed tomography scans: correlation of radiomics-based features with radiation therapy dose and radiation pneumonitis development. Int J Radiat Oncol Biol Phys. 2015;91(5):1048-1056. doi:10.1016/j.ijrobp.2014.11.030

22. Chaudhuri AA, Binkley MS, Rigdon J, et al. Pre-treatment nontarget lung FDG-PET uptake predicts symptomatic radiation pneumonitis following stereotactic ablative radiotherapy (SABR). Radiother Oncol. 2016;119(3):454-460. doi:10.1016/j.radonc.2016. 05.007

23. Castillo R, Pham N, Castillo E, et al. Pre-radiation therapy fluorine 18 fluorodeoxyglucose PET helps identify patients with esophageal cancer at high risk for radiation pneumonitis. Radiology. 2015;275 (3):822-831. doi:10.1148/radiol.14140457

24. Martel MK, Ten Haken RK, Hazuka MB, Turrisi AT, Fraass BA, Lichter AS. Dose-volume histogram and 3-D treatment planning evaluation of patients with pneumonitis. Int J Radiat Oncol Biol Phys. 1994;28(3):575-581. doi:10.1016/0360-3016(94)90181-3

25. Marks LB, Yorke ED, Jackson A, et al. Use of normal tissue complication probability models in the clinic. Int $J$ Radiat Oncol Biol Phys. 2010;76(3 Suppl):S10-19. doi:10.1016/j.ijrobp.2009.07.1754

26. Seppenwoolde Y, De Jaeger K, Lebesque JV. In regard to Tsujino et al.: predictive value of dose-volume histogram parameters for predicting radiation pneumonitis after concurrent chemoradiation for lung cancer. IJROBP 2003;55:110-115. Int J Radiat Oncol Biol Phys. 2003;56(4):1208-1209; author reply 1209. doi:10.1016/ S0360-3016(03)00345-6

27. Tsujino K, Hirota S, Endo M, et al. Predictive value of dose-volume histogram parameters for predicting radiation pneumonitis after concurrent chemoradiation for lung cancer. Int J Radiat Oncol Biol Phys. 2003;55(1):110-115. doi:10.1016/S0360-3016(02)03807-5

28. Vogelius IR, Bentzen SM. A literature-based meta-analysis of clinical risk factors for development of radiation induced pneumonitis. Acta Oncol. 2012;51(8):975-983. doi:10.3109/0284186X. 2012.718093

29. Cremonesi M, Garibaldi C, Timmerman R, et al. Interim (18)F-FDG$\mathrm{PET} / \mathrm{CT}$ during chemo-radiotherapy in the management of oesophageal cancer patients. A systematic review. Radiother Oncol. 2017;125 (2):200-212. doi:10.1016/j.radonc.2017.09.022 
30. Zhang Y, Yu Y, Yu J, Fu Z, Liu T, Guo S. (18)FDG PET-CT standardized uptake value for the prediction of radiation pneumonitis in patients with lung cancer receiving radiotherapy. Oncol Lett. 2015;10(5):2909-2914. doi: 10.3892/ol.2015.3207

31. Lee HJ Jr., Zeng J, Vesselle HJ, Patel SA, Rengan R, Bowen SR. Correlation of functional lung heterogeneity and dosimetry to radiation pneumonitis using perfusion SPECT/CT and FDG PET/CT imaging. Int J Radiat Oncol Biol Phys. 2018;102(4):1255-1264. doi:10.1016/j. ijrobp.2018.05.051
32. Demirev AK, Kostadinova ID, Gabrovski IR. (18)F-FDG PET/CT in patients with parenchymal changes attributed to radiation pneumonitis. Mol Imaging Radionucl Ther. 2018;27(3):107-112. doi:10.4274/ mirt.55706

33. Guan F, Geng C, Ma D, et al. RBE model-based biological dose optimization for proton radiobiology studies. Int J Part Ther. 2018;5 (1):160-171. doi:10.14338/IJPT-18-00007.1

\section{Publish your work in this journal}

OncoTargets and Therapy is an international, peer-reviewed, open access journal focusing on the pathological basis of all cancers, potential targets for therapy and treatment protocols employed to improve the management of cancer patients. The journal also focuses on the impact of management programs and new therapeutic

Submit your manuscript here: https://www.dovepress.com/oncotargets-and-therapy-journal agents and protocols on patient perspectives such as quality of life, adherence and satisfaction. The manuscript management system is completely online and includes a very quick and fair peer-review system, which is all easy to use. Visit http://www.dovepress.com/ testimonials.php to read real quotes from published authors. 